\title{
Kessel reporter : de la chose vue à la chose lue
}

\author{
Jonathan Barkate
}

\section{(2) OpenEdition}

Journals

Édition électronique

URL : http://journals.openedition.org/aes/986

DOI : $10.4000 /$ aes.986

ISSN : 2258-093X

\section{Éditeur}

Laboratoire LISAA

\section{Référence électronique}

Jonathan Barkate, "Kessel reporter : de la chose vue à la chose lue », Arts et Savoirs [En ligne],

8 | 2017, mis en ligne le 19 avril 2017, consulté le 21 avril 2019. URL : http://journals.openedition.org/ aes/986; DOI : 10.4000/aes.986

Ce document a été généré automatiquement le 21 avril 2019

Centre de recherche LISAA (Littératures SAvoirs et Arts) 


\title{
Kessel reporter : de la chose vue à la chose lue
}

\author{
Jonathan Barkate
}

Il nous paraît, en effet, que le reportage fait partie des genres littéraires et qu'il peut devenir un des plus importants d'entre eux. La capacité de saisir intuitivement et instantanément les significations,

l'habileté à regrouper celles-ci pour offrir au lecteur des ensembles synthétiques immédiatement déchiffrables sont les qualités les plus nécessaires au reporter. ${ }^{1}$

1 La carrière journalistique de Joseph Kessel épouse l'histoire de ses contemporains, de 1919 à 1970. La filiation entre le reporter et l'historien remonte à Hérodote qui, dans Historíai - traduit tantôt par Enquête tantôt par Histoires -, définit le rôle de l'histor, dont la base isto signifie à la fois « voir » et « savoir ». Comme l'enquêteur hérodotéen, le reporter est «celui qui sait pour avoir vu $»^{2}$. Mandaté par un journal pour rendre compte d'un événement dont il est témoin en écrivant sur le vif, il doit donc voir vite et bien : «"savoir voir", c'est voir "juste" ". Mais cette conception idéalisée est simpliste car il est fréquent que le reporter écrive d'une traite à son retour un texte que le journal fragmentera ou qu'il retravaille sa "vision première" dans un "texte second " en lui donnant "les apparences d'une progressive découverte de la réalité $»^{4}$. La nécessaire recomposition qu'opère le reportage remet en cause l'idée d'une écriture fondée sur l'immédiateté, idée selon laquelle le réel est invinciblement saisi par l'œil du reporter et simultanément décalqué par sa plume. Ce rapport homologique du reportage au monde, que l'on retrouve dans la définition sartrienne, repose sur l'illusion que l'écriture journalistique exclut le recours aux artifices littéraires pour décrypter l'histoire dont elle rend compte. En outre, si le reporter est bien doté d'un regard particulier, rien ne garantit son infaillibilité puisqu'il pénètre parfois dans des mondes dont il ignore tout avec mission de les découvrir au lecteur. Le métier reposant sur ce paradoxe, les situations opaques sont inévitables. Les reconnaître permet, par contraste, d'accréditer tout ce qui aura été identifié et expliqué. L'ethos du reporter fiable et honnête est consolidé par sa maîtrise 
d'un savoir pratique qui lui permet de débrouiller des univers nouveaux et que Kessel met en œuvre constamment dans Témoin parmi les hommes, l'anthologie de ses meilleurs reportages, parue en six volumes entre 1956 et $1969^{5}$. Le corpus retenu pour cette étude mêle volontairement des textes rédigés dans des conditions différentes, afin de déterminer d'éventuelles constantes dans la perception du réel. Souvent l'écriture a été conditionnée par l'actualité, comme dans "Sinn Fein", qui relate le combat des indépendantistes irlandais contre l'oppression anglaise en $1920^{6}$, ou dans "Les Forgerons du malheur", qui porte sur la campagne présidentielle allemande de 1932 avec la montée inéluctable du parti nazi ${ }^{7}$. Mais le corpus est également composé de deux reportages dont le sujet est détaché de l'histoire immédiate: dans "Les Chasseurs d'esclaves" (1930), Kessel suit la piste des marchands d'esclaves en Abyssinie ${ }^{8}$, et, dans "Unterwelt" (1932), il infiltre la pègre allemande'. À ces reportages en bonne et due forme s'ajoutent "Les Francs-tireurs de Barcelone", reportage improvisé sur l'insurrection catalane de $1934^{10}$, et "Les Mois funestes", collection d'articles écrits sur le front pendant la drôle de guerre ${ }^{11}$. Dans tous ces textes, Kessel met la question du regard au service de l'efficacité narrative en la déclinant à l'envi : puisqu'il découvre des mondes inconnus, on conçoit que parfois il ne sache pas les voir (la plume rend alors compte de la défaillance de l'œil), mais comme le reporter est chargé de décrypter ces univers nouveaux, on attend de lui qu'il sache les voir (la plume écrivant sous la dictée de l'œil), puis qu'il sache les faire voir (en racontant, la plume sert de relai entre l'œil du reporter et celui du lecteur), quitte à ce qu'il feigne de ne pas savoir voir pour faire raconter par un tiers ce qu'il a su ne pas voir (la plume dessine alors un trompe-l'œil).

\section{Ne pas savoir voir}

2 Afin de mettre en valeur sa capacité à débrouiller un réel complexe, le reporter a intérêt à ne pas se montrer infaillible. Sa clairvoyance n'en sera que plus appréciable. C'est pourquoi il n'hésite pas à se représenter dans des situations où le monde lui est resté opaque, tirant un profit narratif de ces moments où il n'a pas su voir mais qu'il sait raconter.

"Sinn Fein" est construit selon les codes du roman policier et du roman d'espionnage : puisque la République irlandaise n'est pas reconnue par Londres, tous les interlocuteurs du reporter sont recherchés et toutes leurs entrevues sont teintées d'une part de secret. Dans ce contexte, Kessel côtoie des initiés dont les pratiques lui paraissent souvent obscures. C'est le cas dans l'article intitulé «La justice du Sinn Fein », dans lequel Arthur Griffith, le président de la République irlandaise clandestine, démasque un espion des services secrets anglais. Le reporter a beau remarquer que ses voisins ressemblent à des juges, il se méprend sur le sens de la scène et induit donc le lecteur en erreur en ne lui racontant que ce qu'il n'a pas su voir sur le moment. Tout comme lui, le lecteur s'attend à ce que le tribunal condamne le traitre à être exécuté mais il est laissé libre et l'article s'achève sur un coup de théatre lorsque Griffith révèle que ce procès n'était qu'un leurre destiné à confondre les Britanniques devant les journalistes étrangers présents dans la salle - que le lecteur, à la suite du journaliste, avait pris pour des juges. La fausse piste qui a présidé à l'écriture de l'article reprend l'invariant du reportage prétendument écrit sur le vif alors qu'au moment de l'écriture Kessel a connaissance de la révélation finale. En représentant son manque de pénétration, le reporter qui ignore qu'il est entouré de confrères ménage le quiproquo orchestré par Griffith et rend plus frappante la révélation 
dont le rôle est de créer un effet de chute. Une fois averti de la supercherie, le lecteur est à même de relever les indices qu'il avait négligés d'abord et il note que les hommes auxquels Kessel prête « une attitude professionnelle de juge $»^{12}$ sont tous muets, comme le reporter français à qui on a demandé de garder le silence, en sorte qu'il n'a pas su voir qu'il était lui-même l'un de ces juges putatifs à qui Griffith faisait tenir un rôle à leur insu, afin de soutenir son stratagème.

Cette efficacité narrative repose en partie sur le rôle des adjuvants, sans lesquels le reportage n'aurait pas été possible. C'est que l'observateur professionnel est aussi parfois un simple touriste perdu en terre étrangère. Ainsi "Sinn Fein" ne commence-t-il réellement que lorsque Kessel est chaperonné par un guide qui le met sur la piste des insaisissables rebelles. Avant cela, son travail se borne à une errance irritante et stérile, comme il s'en plaint au début du premier article :

Voilà vingt-quatre heures que j'avais atteint Dublin [...] et je n'avais encore rien vu, rien trouvé, qui fût pour mon reportage d'un intérêt véritable. [...] Les jaunting cars, « fiacres » où l'on s'asseyait en plein vent, de côté, les jambes pendantes, et l'accent irlandais, le "brogue », vous dépaysaient complètement. [...] Mais tout cela n'était que plaisirs de touriste. ${ }^{13}$

La disponibilité sensorielle du reporter est comptée pour rien puisque ce qu'il voit et ce qu'il entend de neuf est dépourvu d'intérêt pour son travail. Tant qu'il n'est pas sur une piste sérieuse, il se désole de n'être qu'un touriste parmi d'autres et tout ce dont il est témoin est invalidé par la formule définitive: «je n'avais encore rien vu». Ce dépaysement a les mêmes conséquences dans chaque nouvel univers, en particulier lorsque le reporter découvre un monde aussi secret que celui du trafic d'esclaves. Quand il rencontre un ancien marchand à Addis-Abeba, Kessel s'étonne que l'ordonnance du négus censée avoir mis fin à l'esclavage n'y soit pas respectée. Aziz lui révèle alors ce qu'il n'a pas su voir :

[...] chaque chef ici [...] possède des esclaves. [...] Regardez-les sur leurs mulets de par la ville. Derrière trottent à pied les Abyssins purs, les guerriers, puis les chassemouches, les valets d'étrier, les porteurs, les messagers. Voyez l'attitude de ces derniers. Est-elle pareille à celle d'hommes libres ? ${ }^{14}$

6 Ce décryptage doit permettre au néophyte de se repérer mais toutes les réalités ne sont pas aussi visibles, comme Kessel le remarque en brossant le portrait d'un autre trafiquant :

Saïd nous regardait en souriant de toutes ses dents très blanches. Il portait une courte barbe qui lui donnait un air mâle sans dureté. Il était vigoureux, bien pris dans une ceinture où brillait le manche d'un poignard. Sa mine était fière et intelligente. Jamais je ne l'eusse pris pour un marchand d'esclaves. ${ }^{15}$

7 La dernière phrase, en même temps qu'elle suggère que le secret entoure l'activité de Saïd, déconstruit la vision naïve et préconçue de l'occidental qui imagine le vendeur d'esclaves comme une brute cynique aisément reconnaissable. De même, Kessel se méprend en cherchant à identifier des esclaves. Alors que Saïd l'a mené dans le village où se trouve son entrepôt, le reporter lui demande si les premiers hommes qu'il voit sont des esclaves et s'entend répondre : «Il ne faut pas en voir partout ${ }^{16}$. Encore une fois Kessel est victime d'un manque de clairvoyance : la première fois, il ne reconnaissait pas les esclaves qu'il avait effectivement sous les yeux et la seconde il les invente à tort. Ce n'est qu'après en avoir fréquentés auprès de ses guides que le reporter sera capable de les reconnaître à coup sûr, sachant regarder ce qu'il faut voir. Tant qu'il ne sait pas quoi regarder, il ne sait pas voir. 
8 La défaillance peut également tenir à un problème d'interprétation. Dans "Les Francstireurs de Barcelone", un phénomène a échappé à la sagacité de Kessel qui n'en dit pourtant rien : il est certes acceptable de se montrer faillible, mais il ne convient pas de se représenter totalement imperméable aux événements. Dans la mesure où le lecteur attend du reporter qu'il lui éclaire le monde, chaque fois que son ignorance ne servira pas le récit, elle sera tue. Voici comment Kessel décrit une scène de fusillade menée par les desperados cachés sur les toits :

[...] une stridence de frelon métallique rasa mon épaule et m'arrêta net. À trois mètres de moi, un morceau de plâtre se détacha du mur de l'ancienne maison des chanoines. [...] Il y avait encore des francs-tireurs. [...] Je [...] me renfonçai dans une encoignure. De là, je pus suivre le rythme de la guérilla, la manœuvre des francstireurs.

Leur jeu était facile à saisir. Ils ne se servaient pas de carabines, dont le canon les eût trop aisément trahis. Invisibles, couchés, aplatis sur les toitures, ils étendaient le bras jusqu'au bord et là, fléchissant le poignet dans la direction d'un piquet de garde ou d'une patrouille, lâchaient leur coup de pistolet au jugé, sans viser. ${ }^{17}$

Ce morceau de bravoure représentant le reporter en baroudeur intrépide met en valeur son courage, sa perspicacité et son instinct professionnel car c'est en restant exposé au danger que Kessel peut voir et comprendre la scène, comme il l'affirme crânement dans la formule « [1] eur jeu était facile à saisir ». Or, cette clairvoyance est tout à fait artificielle, comme le révèle, trente ans après le reportage, la préface de Pour l'honneur, dans laquelle est présentée Une Balle perdue, la nouvelle adaptée des "Francs-tireurs de Barcelone" :

Aplati contre les pierres vénérables, réfugié tantôt dans un cloître de couvent, tantôt sous un porche de palais antique, j'essayais en vain de comprendre. Un journaliste espagnol, que je rencontrai dans l'un de ces abris, m'éclaira enfin. Il ne s'agissait pas d'une nouvelle révolte, mais seulement de quelques francs-tireurs dispersés sur les toits. ${ }^{18}$

10 En admettant tardivement, hors du reportage, qu'il ignorait tout de la guérilla espagnole et que le sens de la scène lui échappait totalement, Kessel montre qu'un reporter n'avoue qu'avec parcimonie avoir manqué de clairvoyance afin de ne perdre ni la confiance ni l'estime de son lecteur. S'il lui est permis de ne pas savoir voir, c'est uniquement au seuil du monde qu'il découvre ou très ponctuellement si le récit le demande. Une fois qu'il a pénétré un monde, il doit le révéler au public, fût-ce en étant guidé par des adjuvants, selon un schéma narratif bien connu. Chargé d'une quête, le reporter est soumis à des épreuves initiatiques qu'il doit surmonter pour accéder à la vérité. Son travail le rend digne de remplir cette mission car le reporter est un élu qui, tel un héros de conte, est doté d'un pouvoir : celui de savoir voir.

\section{Savoir voir}

11 L'acuité du regard du reporter s'explique avant tout par sa disponibilité sensorielle et les détails apparemment anecdotiques qui jalonnent ses articles renforcent l'impression de réalité. Même lorsque Kessel se lamente de n'avoir rien vu depuis son arrivée à Dublin, il se représente en train d'observer : «Par la fenêtre de la chambre que j'avais prise à l'hôtel Shelbourne, je regardais l'inépuisable bruine irlandaise envelopper la ville ${ }^{19}$. Il n'est pas si anodin qu'il soit posté à un lieu d'observation et qu'il évoque ce qu'il voit, même dans sa détresse, à laquelle s'accorde la tristesse de la bruine. Ce qu'indiquent ces détails, c'est que Kessel est préparé à voir ce qu'il ne voit pas encore : parce qu'il reste en alerte, le 
reporter va pouvoir réagir dès qu'on l'aura contacté pour le mettre sur la piste des combattants irlandais.

Une fois en chasse, sa première qualité est de se ménager de bonnes conditions d'observation, au plus près de l'action, comme lors de la fusillade barcelonaise. Kessel explique que ce savoir-faire est lié à une volonté farouche inhérente au métier lorsqu'il se remémore la drôle de guerre en déplorant que les journalistes aient été contraints de suivre l'armée sans avoir eu la liberté d'aller où bon leur semblait sur le front : « chacun de nous attendait avec impatience l'instant de quitter ces circuits collectifs pour voir, à l'avant et de près, cette étrange guerre d'attente et les soldats qui la faisaient $»^{20}$. L'ardent désir des journalistes est mis en valeur par l'ordre des mots qui a pour effet de laisser croire que le verbe voir est employé sans complément. Cet exemple est comparable à l'emploi absolu du verbe savoir dans la présentation de l'article sur Rethel alors que le travail des journalistes est toujours entravé après le début de l'offensive allemande: "Que pesaient, en des instants pareils, les sanctions dont je pouvais encourir les foudres? Ce qu'il fallait avant tout, c'était savoir ${ }^{21}$. Kessel n'écrit plus qu'en son nom propre : il ne s'agit plus d'une aspiration commune à tous ses confrères, mais d'un risque qu'il assume seul. Le verbe savoir exprime sa volonté de voir et les deux verbes sont équivalents: si le reporter voit, alors il sait ; s'il sait, c'est qu'il a vu.

13 Lorsqu'il est libre de ses mouvements et qu'il ne fait pas de long voyage, Kessel se fait accréditer pour assister aux grands événements de manière à les suivre au mieux, comme lors d'un discours de Hitler pendant la campagne présidentielle de 1932 :

Je n'eus aucune peine [...] à me trouver au premier rang du parterre, tandis que, derrière moi, et tout autour, étagée sur les gradins de l'amphithéâtre géant, se pressait une foule compacte, immense. ${ }^{22}$

La mise en scène de soi du reporter révèle comment il a opéré pour être si bien placé. Ce n'est que dans un second temps que Kessel raconte ce qu'il a vu, en tirant parti de sa position privilégiée :

Section par section, défilaient des rachitiques, des corps déformés, des visages d'anormaux... Toute cette adolescence était flétrie, vieillie, rongée par une atroce débilité physique et morale. Il semblait qu'elle sortît d'un hôpital, d'un hospice, d'un asile. [...] Mais des gradins, des rangs éloignés du parterre, on ne pouvait distinguer cela et les ovations accompagnèrent le déroulement de ces cohortes spectrales. ${ }^{23}$

Le contraste entre la faiblesse des troupes et la démonstration de force que constitue ce défilé est redoublé par la différence de perception selon le rang de chacun dans la tribune. Le reporter se distingue de la foule parce qu'il a su se placer pour voir ce qu'elle ne peut discerner et, de ce fait, lui seul est à même de développer un point de vue critique sur ce qu'il voit. Sa clairvoyance politique, perception intellectuelle, repose donc sur une bonne perception physique. Même quand il n'est pas en reportage commandé, Kessel sait tirer parti du hasard pour s'offrir une position stratégique, comme la fenêtre de sa chambre d'hôtel à Barcelone, qu'il qualifie d'« observatoire exceptionnel » ${ }^{24}$ parce qu'elle lui offre une vue surplombante qui lui permet de comprendre qui tient la ville et comment chaque faction réagit. Kessel se représente, physiquement et symboliquement, au-dessus des hommes : il a su voir - et déduire ce qu'il n'avait pas vu - parce qu'il était placé en hauteur et parce que cette position marque la primauté de son regard de reporter. La transposition romanesque confirme cette supériorité puisque, dans la nouvelle tirée du reportage, l'étrangère qui suit l'insurrection depuis la fenêtre de sa chambre d'hôtel est désavouée par sa fin tragique: Helen Moore meurt d'une balle 
apparemment perdue mais en réalité tirée sciemment par Alejandro, le personnage principal, qui, après l'avoir admirée, la punit d'avoir regardé ses camarades mourir avec indifférence, un verre à la main, comme si elle assistait à un spectacle depuis une loge de théâtre. Le personnage qui ne sait que regarder passivement paie son incapacité à voir, ce dont est préservé le reporter, qui sait voir et ne se contente pas de regarder. Le choix du romancier éclaire ici la conception du reporter, dont la vision est conditionnée par l'engagement dans l'action de voir.

La bonne perception de Kessel ne dépend toutefois pas uniquement des conditions d'observation qu'il n'a pas toujours le loisir de se ménager. Il lui faut donc développer un talent qui relève moins de la technique que de l'instinct car il doit voir vite et juste. L'urgence est consubstantielle à son métier mais la vitesse ne doit pas obérer la précision de sa vision. Aussi le reporter qui sait voir est-il doué de discernement. Savoir voir, c'est également savoir qu'il y aura à voir. Lorsqu'il décide d'assister à une réunion de quartier du parti nazi, Kessel devance l'événement : «Les forgerons de Hitler travaillaient cette nuit-là, entre autres lieux, à Reinickendorf. L'endroit me parut le mieux choisi pour les surprendre à l'ouvrage $»^{25}$. L'anticipation du reporter est marquée par l'emploi des verbes paraitre et surprendre qui exprime son analyse préalable de la situation que la vision physique ne fait que confirmer : sachant ce qu'il veut trouver, il sait aussi où le chercher. De là naît une sélection des informations, mais il arrive que la hiérarchisation apparaisse dans le texte, comme dans "Les Chasseurs d'esclaves" :

Je pourrais dire aussi, et longuement, comme dans un étrange conte d'aventures, les marches au clair de lune, les campements à la belle étoile, le feu de broussailles qu'on allume entre trois pierres, les pistes perdues, retrouvées, l'ivresse de la fatigue et cet étonnant bien-être physique qui permet, après dix heures de route, après un repas fait de riz et d'eau trouble, après un sommeil sur un lit de cailloux, de se réveiller à l'aube sans une courbature et de repartir avec joie vers des risques nouveaux.

Mais, en vérité, nous n'avions qu'un seul but, un seul dessein, et que le soleil, le désert, la fatigue portaient jusqu'à l'obsession : rejoindre la caravane d'esclaves. ${ }^{26}$

Le contraste de longueur entre les deux paragraphes met d'autant mieux en valeur l'effet de chute qu'il succède à une accumulation d'où ressort l'urgence, sensible à la fois dans la hâte exprimée par Kessel et dans le rythme de la période au conditionnel. La hiérarchisation des informations est biaisée puisque le volume textuel dévolu à ce que Kessel ne raconte pas est bien plus important que celui réservé à ce qui, seul, dit-il, occupe ses pensées. Ces évocations, sous forme de prétérition, sont autant de scènes à faire que le reporter fait mine de ne pas pouvoir se permettre, tenu qu'il est par une urgence étrangère au littérateur.

En choisissant ce qu'il convient de développer, Kessel déploie un éventail «allant du regard qui photographie au regard qui perce $»^{27}$ dont la différence de degré affleure dans le portrait de Hitler. D'abord assez neutre, la description physique est d'ordre photographique :

Devant moi se tenait un homme vêtu d'un médiocre costume noir, sans élégance, ni puissance, ni charme, un homme quelconque, triste et assez vulgaire. Une raie soigneuse sur le côté partageait ses cheveux plats et ternes. Il avait le front plat, le nez court et relevé en l'air par une pointe aiguë, les joues étaient roses. Au-dessus d'une toute petite bouche une moustache réduite à une tache noire semblait l'effet d'un comique laborieux.28

L'homme suinte la médiocrité et le ridicule, mais son portrait est infléchi lorsqu'il prend la parole : 
[...] assuré de son magnétisme, [Hitler] se lança dans un délire de mégalomane.

Seulement, ce délire, il le conduisait avec l'allure du maître, du dominateur dont l'Allemagne éperdue avait besoin. Cette allure allait déplorablement à son physique; mais comment pouvaient-ils s'en apercevoir les milliers d'hommes [...] qui l'avaient attendu comme un sauveur ? ${ }^{29}$ décrypter ce qui relève de l'impalpable, comme en témoigne l'accumulation de substantifs abstraits exprimant à la fois le dangereux sens politique de Hitler et la fascination qu'il exerce sur la foule. Cette gradation du regard qui photographie au regard qui perce signale en outre la capacité à enregistrer la réalité avant de la déchiffrer, à pressentir avant de voir. Doté d'une faculté d'anticipation, le reporter est également doué d'un sixième sens qui est une sorte de prescience, une vision d'avant la vue. Ce « troisième œil $\aleph^{30}$, Kessel l'exerce dans les milieux qu'il découvre. Alors qu'il redoute que ses deux contacts de la pègre allemande se fassent remarquer dans le hall de son hôtel, il n'en est rien, parce qu'inconsciemment il a su choisir des guides qui lui correspondraient :

Je compris alors l'instinct qui, la veille, dans ce bar louche, m'avait poussé vers eux :

c'étaient deux aristocrates parmi leurs confrères à face brutale, au corps grossier.

C'étaient des princes de la pègre. ${ }^{31}$

La part faite à l'instinct confère à Kessel une intuition qui le rend capable de reconnaître les êtres d'élection. Son sens de la nature humaine est si développé qu'il juge ses semblables avec acuité au premier coup d'œil, avant même que le regard se soit montré perçant. Chez lui, le regard qui photographie a déjà la capacité de dépasser les apparences pour approcher la réalité des êtres et des choses.

En somme, le reporter qui sait voir se montre clairvoyant aussi bien en remarquant des détails ou en les anticipant par instinct ou par réflexe professionnel qu'en reconnaissant et en analysant des événements importants. Dans "Les Francs-tireurs de Barcelone", Kessel prédit immédiatement l'inanité de la terne insurrection dont il est témoin :

Des éclairs de magnésium [...] me permirent de surprendre les visages des auditeurs. Et, du coup, il me fut impossible de croire que je vivais un moment de grandeur. [...] je ne vis autour de moi que plate curiosité, attente passive et molle réaction. [...] Où était la ferveur farouche et silencieuse des sinn feiners de l'Irlande héroïque ? Où les masses délirant sous l'hystérie calculée de Hitler et ses cohortes brutes aux visages de meurtre ?32

La lucidité politique de Kessel s'explique par son expérience de la ferveur populaire: parce qu'il a déjà vu des événements historiques, il est capable de les discerner et de distinguer un soulèvement anodin voué à l'échec d'« une révolution véritable »33. Le reporter - Kessel, tout du moins - est donc un être à part, dont la pénétration s'oppose à l'aveuglement des hommes plongés dans l'événement qu'ils vivent. Par sa clairvoyance, il doit déciller les yeux de ses contemporains mais savoir voir n'est pas une fin en soi. C'est aussi un moyen de savoir raconter : puisqu'il doit informer et donner à imaginer, c'est-àdire faire savoir et faire voir, le reporter doit savoir faire voir.

\section{Savoir faire voir}

Pour faire de la chose vue une chose lue, il faut que le reporter éclaire la réalité qu'il a observée de son point de vue singulier car il n'est pas soumis à l'objectivité qu'on lui prête : 
Pour que la réalité baigne dans sa couleur originelle, il faut donc la retremper dans la littérature. [...] Pas de grandes visions, donc, sans [...] un texte "écrit ", où compte le talent du journaliste en tant qu'auteur ${ }^{34}$.

Paradoxalement, l'authenticité de la vision réside dans l'artifice littéraire qui lui confère sa dimension narrative. C'est ce qui explique que Kessel se mette en scène, quitte à produire «un effet de fictionnalisation a posteriori $»^{35}$ à force de multiplier les protestations de sincérité, comme dans "Unterwelt" où la surenchère permanente dans l'incroyable - incroyable mais vrai - est un motif structurant, dès la préface rédigée à l'occasion de la reprise du reportage dans Témoin parmi les hommes:

Quand je me souviens aujourd'hui des endroits que j'ai traversés, des hommes que j'ai côtoyés, des lois qui les régissaient, je doute de mes sens et de ma raison.

Pourtant, les articles sont là, que je téléphonais au Matin dans les journées mêmes où je faisais ces incroyables découvertes. ${ }^{36}$

De telles formules sensationnalistes sont récurrentes au sein du reportage. L'article augural en comporte une première après qu'un malfrat a expliqué l'attachement des hors-la-loi à la discipline :

Je n'invente rien, je ne fais pas de rapprochement forcé. Je reproduis les paroles textuelles d'un des princes du monde souterrain qui vit des charmes d'une femme d'un établissement de nuit, ne dédaigne pas le cambriolage et a fait plusieurs années de prison. ${ }^{37}$

Kessel insiste sur le paradoxe qui donne son titre à l'article - « La loi des hors-la-loi » - et qui consiste, pour un délinquant qui viole les règles de la société, à respecter scrupuleusement celles qui régissent l'Unterwelt. Introduit dans ce monde qui renverse les valeurs communes, le reporter conserve sa circonspection habituelle mais il constate qu'elle est superflue lorsqu'il énonce la seconde caution d'authenticité : «Je pensais qu'il exagérait la puissance de l'Unterwelt. Les soirées qui suivirent devaient m'enlever toute incrédulité ${ }^{38}$. Pareille formule permet de représenter un univers étonnant et de jouer sur les conventions narratives pour exacerber le caractère sensationnel de la découverte, puisque la prolepse contenue dans la dernière phrase amène à anticiper les surprises que réserve la suite du récit.

Afin d'éviter que de tels passages incitent le lecteur à douter de ce qu'il lit, Kessel se représente au travail et met en scène la matérialité de son enquête. Ainsi le premier article d'“Unterwelt" est-il la retranscription d'un entretien que le reporter mène avec ses guides et, s'il donne largement la parole aux truands, il précise qu'il n'a pas renoncé aux fondements déontologiques de son métier : «il me faut résumer les informations que je reçus ce jour-là, et dont j'ai contrôlé l'exactitude par des recoupements précis »39. Plus l'objet du reportage menace de n'être pas cru, plus le reporter affirme avoir été rigoureux dans ses vérifications. En Abyssinie, Kessel s'astreint à la prudence puisque l'esclavage étant officiellement interdit, il ne peut espérer être mis sur une piste que s'il garde secret le but de sa mission, ce qui ne va pas sans difficulté car une « seule question imprudente pouvait non seulement [lui] fermer toutes les portes, mais encore [le] faire expulser $»^{40}$. Le reporter rend palpable la contrainte qui préside à sa discrétion dans une scène où il se met en quête d'un trafiquant d'esclaves :

Je me dépensai en démarches sournoises, en questions d'apparence innocentes et en détours variés pour joindre [Saïd]. Tous mes efforts furent vains. Non point qu'il se cachât, mais il était inconnu des Européens. Quant aux indigènes, son nom ne leur apprenait rien si je n'y ajoutais pas sa profession. 
Mais je voulais la taire à tout prix. Non seulement il était indispensable qu'on ignorât l'objet de mon enquête, mais encore et surtout je n'avais pas le droit de compromettre un homme à qui j'étais envoyé en ami. ${ }^{41}$

de notes tend à invalider dans un autre contexte. L'image de baroudeur de Kessel ne s'accommode guère en effet du carnet qu'on noircit car il rompt plutôt l'élan de l'aventurier. Mais dans une salle hostile, fanatisée par un discours revanchard et nationaliste, prendre des notes témoigne de l'intrépidité du journaliste français face à la menace de la foule. Ce passage met également en valeur la faculté qu'a le reporter de se représenter dans les yeux des autres. Pour qui prétend voir et montrer, il est capital de déplacer son regard car "voir, c'est aussi être vu par l'autre »" Kessel opère régulièrement ce décentrement pour donner un surcroît de sens à ce qu'il décrit. L'avilissement des esclaves est ainsi exacerbé par le regard morne qu'ils posent sur le reporter :

Les yeux des esclaves étaient fixés sur nous. Je n'en ai jamais croisé qui fussent les truchements d'une pensée aussi rudimentaire. Seuls des yeux de ruminants doux, peureux et passifs, peuvent donner une idée de l'expression qu'avaient les grosses prunelles roulant à fleur du visage. ${ }^{44}$

31 Le portrait de ces êtres déshumanisés au regard bovin repose sur un détail physique qui révèle leur atonie et la comparaison animale montre qu'ils sont aussi domestiqués que les bêtes les plus placides. Le rapport de supériorité est inversé en Allemagne, où les aristocrates du Herren Klub qui soutiennent la candidature de Hindenburg font sentir au reporter qu'ils évoluent dans un autre monde : "chaque tête se tournait vers moi d'un bref et sec mouvement. Et le regard, en général vitrifié par un monocle, me toisait avec indifférence et hauteur $»^{45}$. En décentrant ainsi le regard et en se plaçant dans l'œil de ses interlocuteurs, Kessel permet au lecteur de s'identifier à lui et d'éprouver ce qu'il a ressenti face aux esclaves pathétiques et aux junkers méprisants. De la sorte, la chose vue est associée à la chose vécue et cette alliance donne au lecteur l'impression de partager l'expérience du reporter. Le phénomène d'identification est renforcé par la « poétique du franchissement $»^{46}$, qui confère au reportage une dimension romanesque imitée du conte ou du roman d'apprentissage. Le journaliste passe en effet des frontières en acquittant des tributs qui varient selon le contexte. L'accès à une réunion du parti nazi se fait en échange de monnaie sonnante et trébuchante, mais le droit d'entrée ne garantit pas au reporter qu'il pourra ressortir de la salle :

Ils me laissèrent passer après que j'eus payé les 30 pfennigs d'entrée. Mais j'avais été obligé de prononcer quelques mots. Mon allemand est peu sûr, on me reconnut pour un Français. Dès ce moment, et jusqu'à la fin de la réunion, des regards durs et pesants ne me quittèrent plus. ${ }^{47}$ 
Le franchissement repose ici sur une transgression d'ordre mythique dans la mesure où le reporter - et le lecteur à sa suite - vit une catabase qu'exprime déjà le substantif Unterwelt , qui signifie «monde souterrain ». Kessel joue donc de la gradation initiatique avec gourmandise dans des accumulations assorties de répétitions et de comparatifs comme : « les caveaux succédaient aux caveaux, les cellules s'ajoutaient aux cellules toujours plus étroites, plus sourdes, plus oppressantes $»^{49}$. Deux pages plus loin, il conclut sur le même ton : «C'était vraiment un monde souterrain qui, de cave en cave, de bouge en bouge, de coupe-gorge en coupe-gorge, étendait ses ramifications sous le sol de Berlin ${ }^{50}$. Dans ce « véritable dédale " $^{51}$, l'intertexte mythologique - signalé par l'antonomase créée à partir $\mathrm{du}$ nom du père d'Icare et par le thème de la descente aux enfers - facilite la représentation et transforme la chose vue et vécue par le reporter en chose vue et vécue par le lecteur familier de Virgile, d'Ovide ou de Dante. Par cet effet de crescendo, le reporter entend montrer qu'il dévoile le monde de plus en plus précisément à mesure qu'il descend dans les arcanes des bas-fonds. Gagnant en précision, l'écriture se fait picturale et le reportage devient tableau vivant. C'est toujours sa double dimension visuelle et allégorique qui fait d'un morceau de bravoure une fresque, que ce soit le tableau d'une veillée funèbre en Irlande ${ }^{52}$ ou celui de la curée menée par des esclaves sur un bœuf fraîchement tués3. La force évocatrice de tels passages repose souvent sur une hypotypose. Dans l'article décrivant les ravages du sac de Balbriggan mené par les troupes anglaises, Kessel construit la vision d'horreur pour la rendre la plus expressive possible et, afin que le lecteur français de 1920 se représente l'ampleur du désastre, il emploie une analogie avec les villes dévastées par les bombardements de la Grande Guerre :

Comment traduire cette impression de dédoublement subit? Je savais que j'étais en Irlande et dans l'automne 1920. Mais il me semblait en même temps que j'étais ramené à trois années en arrière et que je me trouvais en Picardie, Flandre ou Champagne, bref sur toute cette aire du front que la guerre frappait, ravageait. [...] C'étaient les mêmes pans de murs effondrés sur des meubles couverts de plâtras, le même arrêt d'une humble vie commune, et son intimité dévoilée soudain et flétrie. Les mêmes chambres sans toit. Les mêmes toits sans support. Les mêmes poutres calcinées, pareilles à des moignons. Les mêmes ferrailles tordues et comme suppliciées, squelettes des maisons, débris fumants, odeur d'incendie, odeur de mort. Tout était pareil. Et pareils aussi les visages des habitants avec leur misère, leur douleur, leur effroi, leur hébétude. ${ }^{54}$

Outre l'analogie entre une paisible bourgade et un théâtre de guerre, la construction de la vision repose sur l'anaphore qui prend en charge les détails de la description et la porte à un degré d'intensité tel que le lecteur a l'impression de voir et de sentir les débris fumants comme s'il était sur les lieux. Ce déploiement de l'hypotypose témoigne de la maîtrise du récit de Kessel grâce à laquelle la chose vue est donnée à voir en même temps qu'elle est transformée en chose lue. Savoir faire voir ne se limite cependant pas à combiner un savoir voir et un savoir raconter. Le reporter doit aussi savoir faire raconter et, pour cela, il convient qu'il se représente ne sachant pas voir. 


\section{Savoir ne pas voir}

35

reportage: parce qu'il a rencontré Monfreid, Kessel bénéficie de ses connaissances et, grâce à lui, rencontre Aziz, le vieux marchand enrichi, puis Saïd. Cette assistance « de différents Charons successifs $\|^{56}$ est un autre invariant du conte transposé dans le reportage car ces adjuvants aident le reporter à réussir sa quête, dont il destine le fruit au lecteur. Le reportage insiste généralement sur cet élément du schéma actantiel : dans "Sinn Fein", Kessel est pris en charge par le réseau des partisans irlandais, du garçon d'étage de son hôtel au président de la République, Arthur Griffith, et dans "Unterwelt", les clés du milieu lui sont livrées par ses deux guides. Même dans des reportages où il travaille seul, Kessel bénéficie ponctuellement d'une aide extérieure. C'est le cas dans "Les Forgerons du malheur", où il s'étonne de l'indifférence de la presse et de la population après la mort d'un homme dans une énième bataille de rue entre hitlériens et communistes :

Le partisan de Hitler avait été tué dans une rue ouvrière, la Chorinerstrasse, devant le numéro 8. Je demandai à un journaliste allemand qui connaissait bien ces parages de m'y accompagner. Il me regarda avec étonnement, mais accepta de me rendre ce service. ${ }^{57}$

Le confrère de Kessel le guide ainsi tout au long de l'article et disparaît dans les suivants mais sa présence aura servi au reporter français à révéler que l'indifférence générale est due à la répétition de tels décès depuis le début de la campagne présidentielle. Cette place de choix réservée aux guides s'explique par la nécessité, pour le reporter, de parvenir à leur degré d'information et de connaissance afin de le partager avec le lecteur. La chaine formée par les intermédiaires dans le texte s'allonge .de deux maillons au niveau métatextuel, puisque le journaliste apprend de ses guides et renseigne ensuite le lecteur. De cette manière, il crée l'illusion que deux mondes se rencontrent grâce à lui : le lecteur français est censé tout savoir de l'Irlande révolutionnaire, des chasseurs d'esclaves ou de la pègre allemande par le truchement de celui qui les lui a fait découvrir.

Kessel affectionne également les révélations progressives et l'effet qu'elles produisent. C'est pourquoi, au lieu de se représenter dans le rôle de celui qui sait déjà, il feint l'ignorance en présentant une scène en deux temps, d'abord telle qu'il l'a vue lui-même son regard se contente alors de photographier - puis telle qu'on la lui a décryptée ensuite - le regard qui perce étant dans ce cas non pas le sien mais celui de son guide. La découverte solitaire de Cork donne ainsi lieu à la description d'une ville bucolique et fiévreuse : 
Étrange cité ! Le long de sa lente rivière, sur laquelle les gouttes de l'averse faisaient courir des vibrations sans fin, elle était irlandaise à l'extrême, avec ses antiques maisons délavées, ses ponts frustes et ses voiliers endormis au balancement des mâts dépouillés. Et, en même temps, les étroites rues en palier accrochées aux pentes des collines ondulées et verdoyantes qui affleuraient au centre de la ville, donnaient à cette partie de Cork la douceur et le calme des vieilles villes italiennes. Mais quelles que fussent les différences d'aspect, la variété physique des quartiers, leur expression spirituelle demeurait partout la même, et c'était une expression de tristesse farouche, de misère violente, de haine désespérée. [...] Cork, ville de grisaille, de pauvreté, de patience pesante, et de sourde menace ! Cork dont, l'année dernière, le lord-maire a été assassiné et dont Terence Mac Swiney, le lord-maire actuel, glisse volontairement vers l'agonie et la mort [...]. Cork, réduit tragique du Sinn Fein. ${ }^{58}$

Ce premier regard est celui du reporter informé du contexte politique mais l'anaphore finale est trop vague pour être fondée sur l'observation concrète de détails révélant la menace qui agite la ville. Ce n'est que grâce à l'œil plus informé d'un conseiller municipal que Kessel remarque les stigmates du conflit illustrant ses réflexions abstraites :

[Aidé] par le bref commentaire de mon guide, je vis Cork sous un visage tout différent, son visage de combat.

Je vis les vitres des boutiques brisées et les devantures remplacées par des planches.

- Coups de crosse... amusement des soldats anglais, disait O'Callaghan.

Je vis sur les murs, pareils à des cicatrices, les éraflures des balles.

- On tire ici sans sommation, disait O'Callaghan. Nous répliquons de la même manière..$^{59}$

Le ton n'est plus à l'évocation poétique mais au témoignage précis et froid. Ni impressions ni sensations mais des choses vues et précisément nommées, comme si le verbe voir, répété à trois reprises, marquait le déchirement du voile devant les yeux de Kessel. Savoir ne pas voir tous ces détails lors de la première visite de la ville permet de les rendre plus scandaleux lorsqu'ils sont évoqués parce qu'ils rompent l'harmonie et la quiétude d'une ville moins faite pour le combat que pour la contemplation. Il est donc narrativement plus intéressant de rejouer ce dévoilement en deux temps que de se présenter comme le garant d'un savoir acquis cherchant à imposer ce qu'il a ressenti. Plutôt que de dicter au lecteur une révolte factice, le reporter choisit de la lui faire éprouver et la portée argumentative du texte n'en est que plus efficace.

41 Les moments où Kessel laisse l'initiative à ses guides sont légion et tous visent à donner de la force au récit. Scène de la vie souterraine :

Après une course à tombeau ouvert, la petite voiture américaine qu'Albert conduisait nous déposa devant un luxueux établissement de nuit. Le chef des chasseurs lui-même vint ouvrir les portières. Comme par hasard, c'était un gaillard formidable et, comme par hasard, il chuchota un mot aux oreilles de Dick, en indiquant d'un signe furtif quatre hommes de mise modeste qui s'entretenaient à la porte du restaurant avec un air béat.

Aussitôt, Albert éprouva le besoin de relever le capot de la voiture et de se pencher sur le moteur, et Dick me prit par le bras d'une façon irrésistible pour me conduire vers l'affiche du cabaret. Planté devant elle, mais les yeux fouillant les alentours, il me dit rapidement :

- Eux - quatre détectives. Dedans - un frère à nous, poursuivi. Le chasseur chef, frère à nous aussi, a reconnu les policiers. Il est allé avertir..$^{60}$

Ce sont ici les malfrats qui savent voir; ils partagent leur perception de la scène avec Kessel qui, lui, n'a su que regarder sans comprendre. Non seulement il n'est pas averti de ce qui fait se déplacer Dick et Albert, mais surtout il ne déchiffre pas la scène. La mention 
objective d'une action visiblement incohérente - «Albert éprouva le besoin de relever le capot de la voiture » - met en valeur son absurdité pour le reporter qui semble ne pas voir que ce geste n'est qu'un prétexte pour justifier auprès des quatre détectives la présence de la voiture devant l'hôtel. Les rares éléments de compréhension de la scène sont donnés au journaliste par Dick et tout le reste est implicite. Le lecteur, une fois en possession de ces informations, est capable de rétablir ce que l'auteur ne lui dit pas et il parait lui aussi surpasser le reporter dans son appréhension de la scène. Le traitement de ce passage est très subtil parce que Kessel feint de n'y rien comprendre tout en mettant en valeur ses guides, qui la lui décryptent, et son lecteur, qui la comprend plus vite que lui. La feintise réside notamment dans l'emploi de l'ironie, lisible dans "comme par hasard ", où l'antiphrase et la répétition expriment la distanciation : rien n'est dû au hasard et si Kessel a su remarquer les signes de connivence entre le chef des chasseurs et l'un de ses guides, il les a également compris puisqu'il en rend compte en alternant le sous-entendu - rien n'est dit, d'abord, de l'identité ni des intentions des quatre hommes, pas plus que de l'urgence qui a causé la course folle - et la lourdeur pédagogique de la répétition de l'expression antiphrastique. De cette façon, le reporter suggère qu'il a su voir les détails qu'il laisse le soin au lecteur d'interpréter à sa place, ce qui témoigne de sa science du récit car il s'efface quand l'efficacité narrative requiert que le texte se passe, apparemment, de sa présence.

Dans les reportages où Kessel ne dépend d'aucun guide, à Barcelone et sur le front, il ne cherche ni à tout voir ni à tout expliquer de lui-même car il n'est pas omniscient. Il se fait raconter ce à quoi il n'a pas assisté, de sorte qu'il devient passeur de témoignages. Le reportage est alors constitué de récits enchâssés où l'événement a été vécu par le témoin et non par le reporter: il relate moins une chose vue qu'une chose racontée. Savoir raconter revient dans ce cas à savoir faire raconter, c'est-à-dire à savoir susciter et recueillir des témoignages. Finalement, le lecteur est bien confronté à une chose vue et vécue mais les marques des témoignages recueillis sont parfois effacées par Kessel qui donne l'impression de parler en son nom. C'est le cas du récit de la bataille de Rethel, qui a pourtant précédé l'arrivée du reporter dans cette ville :

Le bataillon tint le coup. Ni les avions ni les chars ne l'épouvantèrent. Bientôt, un autre bataillon vint se ranger à ses côtés. Puis un régiment. [...] On vit qu'il n'était pas surhumain d'arrêter l'Allemand. [...] La densité du front s'épaissit. Sa puissance de feu s'accrut. [...] Avant-hier, en effet, les Allemands voulurent prendre Rethel. Ils ne ménagèrent ni les moyens, ni les hommes, ni le temps. [...] J'ai pu voir les traces du combat dont l'enjeu était d'une importance capitale et les hommes qui l'avaient mené à bien. Comment les décrire tous ? [...] Au milieu de leurs camarades, une demi-douzaine de garçons racontaient leur histoire. [...] Jamais récit ne fut mené avec tant de naturel, de bonne humeur.

Je serrais les poings en pensant qu'un chauffeur affolé avait failli me priver de l'entendre. ${ }^{61}$

Pendant plus d'une page - sans les coupes - aucun indice ne révèle au lecteur l'identité du narrateur de ce récit. Le reporter prend à son compte les détails du déroulement des opérations alors qu'il a révélé plus tôt dans l'article qu'il était entré dans Rethel après la bataille. L'enchâssement est signalé tardivement et seule la dernière phrase explicite la situation de Kessel, auditeur de ce récit avant que d'en être le passeur. Cette indistinction s'explique par le nombre et l'enthousiasme des témoins, dont le reporter a dû recomposer les histoires pour les rendre fluides et cohérentes. Cet exemple représente le cas extrême du savoir ne pas voir puisque Kessel n'a effectivement pas vu ce qu'il raconte sans que cela l'empêche d'écrire. Mais son discernement, qui s'exerce de bien des façons, le 
conduit ici à repérer la force narrative qu'il peut tirer d'un épisode à reconstituer, ce qui montre son don de double vue : son œil a pour territoire le monde qu'il parcourt au gré de ses enquêtes aussi bien que la page destinée à recréer cet univers pour un lecteur qui ne voyage pas. Savoir ne pas voir, c'est donc être capable de se mettre à la place de ce lecteur sédentaire pour lui donner à voir ce à quoi il n'assiste pas.

Dans sa nécrologie de Kessel, André Lafargue retient deux principaux traits de l'écriture du reporter : «[son] regard qui semblait lointain était d'une rare acuité. Kessel savait voir. Et ce don exceptionnel s'accompagnait, pour notre bonheur, d'une admirable faculté d'évocation $»^{62}$. Orphée découvrant un monde, flâneur au regard acéré ou correspondant de guerre clairvoyant, Kessel a toujours mis la finesse de sa perception au service du récit : chez lui, savoir voir revient à savoir raconter, à savoir faire imaginer afin que le lecteur ait l'illusion de partager la chose vue et la chose vécue. La vision doit donc être magnifiée par l'écriture et la réalité observée être retravaillée à l'aune de la littérature. C'est pourquoi l'œil et la plume du reporter interprètent un ballet dont les différents pas - ne pas savoir voir, savoir voir, savoir faire voir, savoir ne pas voir - ne sont que des variantes consistant à raconter ou à faire raconter en visant la plus grande efficacité narrative possible.

\section{NOTES}

1. Jean-Paul Sartre, Qu'est-ce que la littérature? [1948], Paris, Gallimard, 1975, p. 30.

2. Jacques Fontanille, «Quand le corps témoigne : voir, entendre, sentir et être là (Sémiotique du reportage) ", dans Myriam Boucharenc et Joëlle Deluche (dir.), Littérature et reportage, Limoges, Presses Universitaires de Limoges, 2001, p. 97.

3. Myriam Boucharenc, L'écrivain-reporter au cœur des années trente, Villeneuve d'Ascq, Presses Universitaires du Septentrion, 2004, p. 65.

4. Ibid., p. 151.

5. Par commodité, l'étude a été construite à partir de l'anthologie, plus accessible que les reportages originaux parus dans la presse. Les différences entre les deux états du texte (trois, lorsque le reportage a également été publié en volume avant d'être recueilli dans Témoin parmi les hommes), n'affectent pas la retranscription du dispositif optique dans les articles car les modifications concernent surtout les paragraphes de transition, ajoutés ou supprimés, ainsi que le découpage du texte et le choix des titres, des articles aux chapitres. Tous les reportages seront désignés par le titre qui leur a été donné dans l'anthologie. Ce titre figurera entre guillemets anglais droits, tandis que l'usage des guillemets français s'appliquera au titre des articles composant le reportage (ainsi qu'au titre du tome de Témoin parmi les hommes dans lequel est recueilli le reportage). Ainsi, l'article intitulé «La justice du Sinn Fein » est-il inclus dans le reportage appelé "Sinn Fein", recueilli dans le premier tome de Témoin parmi les hommes, dont le titre est « Le temps de l'espérance ».

6. "Sinn Fein" a paru sous le titre "Chez les Sinn Feiners. L'Irlande révolutionnaire" dans La Liberté, du 9 septembre au 26 octobre 1920.

7. "Les Forgerons du malheur" a paru sous le titre "L'Allemagne aux urnes" dans Le Matin, du 7 au 15 mars 1932. 
8. "Les Chasseurs d'esclaves" a paru sous le titre "Marchés d'esclaves" dans Le Matin, du 26 mai au 14 juin 1930.

9. "Unterwelt" a paru sous le titre "L'Allemagne vue des bas-fonds" dans Le Matin, du 25 mars au 17 avril 1932.

10. "Les Francs-tireurs de Barcelone" a paru sans titre dans Le Matin, du 7 au 14 octobre 1934, puis dans Marianne, sous le titre "Orage sur Barcelone", du 17 octobre au 7 novembre 1934.

11. "Les Mois funestes" recueille une partie des reportages de guerre parus dans Paris-Soir, du 26 octobre 1939 au 8 juin 1940.

12. Joseph Kessel, « La justice du Sinn Fein », "Sinn Fein” [1920], Témoin parmi les hommes, t. I, « Le temps de l'espérance » [1956], Paris, Presses Pocket, 1973, p. 73.

13. Joseph Kessel, « Le ministre traqué », "Sinn Fein", op. cit., p. 37.

14. Joseph Kessel, « Premiers indices ", "Les Chasseurs d'esclaves" [1930], Témoin parmi les hommes ,t. II, « Les jours de l'aventure » [1956], Paris, Tallandier, 2010, p. 18.

15. Joseph Kessel, « Saïd le trafiquant », "Les Chasseurs d'esclaves”, op. cit., p. 37.

16. Joseph Kessel, «L'entrepôt humain », "Les Chasseurs d'esclaves”, op. cit., p. 43.

17. Joseph Kessel, «Les guérilleros », "Les Francs-tireurs de Barcelone” [1934], Témoin parmi les hommes, t. II, « Les jours de l'aventure », op. cit, p. 289.

18. Joseph Kessel, préface de Pour l'honneur [1964], Paris, Le Livre de Poche, 1972, p. 6.

19. Joseph Kessel, «Le ministre traqué », "Sinn Fein", op. cit., p. 37.

20. Joseph Kessel, préface des "Mois funestes" [1939-1940], Témoin parmi les hommes, t. III, «L'heure des châtiments » [1956], Paris, Tallandier, 2010, p. 93.

21. Joseph Kessel, préface de « Rethel ", "Les Mois funestes", op. cit., p. 120.

22. Joseph Kessel, "Les Forgerons du malheur" [1932], Témoin parmi les hommes, t. II, « Les jours de l'aventure ", op. cit., p. 98.

23. Ibid., p. 99.

24. Joseph Kessel, « Grève générale ", “Les Francs-tireurs de Barcelone”, op. cit., p. 258.

25. Joseph Kessel, "Les Forgerons du malheur", op. cit., p. 84-85.

26. Joseph Kessel, «L'aventure », "Les Chasseurs d'esclaves”, op. cit., p. 54-55.

27. Myriam Boucharenc, op. cit., p. 139.

28. Joseph Kessel, "Les Forgerons du malheur”, op. cit., p. 100.

29. Ibid., p. 102.

30. Myriam Boucharenc, op. cit., p. 140-141.

31. Joseph Kessel, « La loi des hors-la-loi », “Unterwelt” [1932], Témoin parmi les hommes, t. II, « Les jours de l'aventure », op. cit., p. 109.

32. Joseph Kessel, "Le balcon de la Généralité ", "Les Francs-tireurs de Barcelone", op. cit., p. 276-277.

33. Ibid., p. 277.

34. Myriam Boucharenc, op. cit., p. 66-67.

35. Renaud Ferreira de Oliveira, "Kessel, Cendrars : deux versions de la pègre ", dans Myriam Boucharenc et Joëlle Deluche (dir.), Littérature et reportage, op. cit., p. 115.

36. Joseph Kessel, préface d'“Unterwelt", op. cit., p. 108.

37. Joseph Kessel, « La loi des hors-la-loi », “Unterwelt”, op. cit., p. 111.

38. Ibid., p. 115.

39. Ibid., p. 112.

40. Joseph Kessel, « Premiers indices ", "Les Chasseurs d'esclaves”, op. cit., p. 17.

41. Joseph Kessel, « Saïd le trafiquant », "Les Chasseurs d'esclaves”, op. cit., p. 36.

42. Joseph Kessel, “Les Forgerons du malheur”, op. cit., p. 87.

43. Myriam Boucharenc, op. cit., p. 141.

44. Joseph Kessel, « Sous le figuier sauvage », “Les Chasseurs d'esclaves”, op. cit., p. 27.

45. Joseph Kessel, “Les Forgerons du malheur”, op. cit., p. 94. 
46. Myriam Boucharenc, op. cit., p. 127.

47. Joseph Kessel, "Les Forgerons du malheur", op. cit., p. 85.

48. Joseph Kessel, « Sésame, ouvre-toi ! ", “Unterwelt”, op. cit., p. 151.

49. Joseph Kessel, « Le royaume des "frères" ", "Unterwelt", op. cit., p. 126.

50. Ibid., p. 128.

51. Ibid., p. 126.

52. Joseph Kessel, « La prière nocturne », “Sinn Fein”, op. cit., p. 88-90.

53. Joseph Kessel, «Sous le figuier sauvage ", "Les Chasseurs d'esclaves”, op. cit., p. 28-29.

54. Joseph Kessel, « Le sac de Balbriggan », "Sinn Fein”, op. cit., p. 94-95.

55. Joseph Kessel, « Premiers indices », "Les Chasseurs d'esclaves”, op. cit., p. 17-18.

56. Renaud Ferreira de Oliveira, article cité, p. 108.

57. Joseph Kessel, "Les Forgerons du malheur”, op. cit., p. 80.

58. Joseph Kessel, « La prière nocturne », "Sinn Fein", op. cit., p. 83-84.

59. Ibid., p. 85.

60. Joseph Kessel, « Le royaume des "frères" ", "Unterwelt", op. cit., p. 121.

61. Joseph Kessel, « Rethel », "Les Mois funestes", op. cit., p. 125-128.

62. André Lafargue, « Kessel : la fin de l'Aventure », Le Parisien, 25 juillet 1979, p. 7.

\section{RÉSUMÉS}

Découvrant des mondes dont il ignore tout, le reporter est chargé de les voir vite et bien pour en rendre compte au lecteur, de sorte que la chose vue se mue en chose lue. L'imbrication de la vision du reporter et de son écriture conduit Kessel à varier les postures pour gagner en efficacité narrative. Que le réel lui soit demeuré opaque ou qu'il ait su le décrypter, le reporter fait savoir et fait voir. Ne pas savoir voir et savoir voir reviennent donc à savoir faire voir en associant à la chose vue la chose vécue. L'emprise du récit est encore plus manifeste lorsque Kessel sait ne pas voir, c'est-à-dire quand il compense son défaut de vision pour rendre compte de scènes qu'il n'a pas comprises ou qu'il n'a même pas vues. Dans tous ces cas, l'écriture du reportage consiste surtout à savoir raconter.

As the reporter discovers worlds he ignores everything about, he has to be quick and good at seeing in order to report them to the reader, so that what he saw turns into what is read. The entanglement of the reporter's vision and of his writing leads Kessel to diversify his positions to gain in narrative efficiency. Whether reality stays opaque or is able to be deciphered, the reporter lets one know or lets one see. By knowing how to see and by not knowing how to see, Kessel demonstrates that he knows how to make one see, through the combination of what is lived and what is seen. The hold of the story is all the more obvious as Kessel knows how not to see, that is to say when he compensates for his lack of vision to report on scenes he did not understand or did not even witness. In all these situations, the essence of writing a report is to tell a story. 
INDEX

Mots-clés : Kessel, reportage, (ne pas) savoir voir, savoir raconter, efficacité narrative

Keywords : Kessel, report, (not) knowing how to see, knowing how to tell, narrative efficiency

\section{AUTEUR}

JONATHAN BARKATE

Université Paris-Est Marne-la-Vallée, LISAA (EA 4120) 